\title{
Dealing with rainfall forecast uncertainties in real-time flood control along the Demer river
}

\author{
Evert Vermuyten $^{1, \mathrm{a}}$, Pieter Meert ${ }^{1}$, Vincent Wolfs $^{1}$ and Patrick Willems ${ }^{1}$ \\ ${ }^{1}$ Hydraulics Division, Department of Civil Engineering, KU Leuven, Kasteelpark Arenberg 40, 3001 Herverlee, Belgium
}

\begin{abstract}
Real-time Model Predictive Control (MPC) of hydraulic structures strongly reduces flood consequences under ideal circumstances. The performance of such flood control may, however, be significantly affected by uncertainties. This research quantifies the influence of rainfall forecast uncertainties and related uncertainties in the catchment rainfall-runoff discharges on the control performance for the Herk river case study in Belgium. To limit the model computational times, a fast conceptual model is applied. It is calibrated to a full hydrodynamic river model. A Reduced Genetic Algorithm is used as optimization method. Next to the analysis of the impact of the rainfall forecast uncertainties on the control performance, a Multiple Model Predictive Control (MMPC) approach is tested to reduce this impact. Results show that the deterministic MPC-RGA outperforms the MMPC and that it is inherently robust against rainfall forecast uncertainties due to its receding horizon strategy.
\end{abstract}

\section{Introduction}

In September 1998, the Demer basin in Belgium incurred a total damage loss of 16 million euros due to flooding [1]. It is no surprise that floods are the natural disasters with the highest economic damage costs. Moreover, the number of floods has strongly increased during the last decades due to two main driving forces: the increasing trend of extreme rainfall events by climate change [2-4] and rising urbanization [5]. The problem of floods will further enlarge in the future, due to these ongoing trends.

Different strategies can be applied to reduce the flood hazard. Few examples are: source control measures such as installing infiltration facilities and detention tanks at large impervious surfaces, installing new retention basins, intelligent real-time control of hydraulic structures. The latter is the strategy studied in this research. It has the advantage to be applicable in densely populated areas lacking space to build new infrastructure by making optimal use of the existing infrastructure.

Model Predictive Control (MPC), or Receding Horizon Control (RHC), was first used in the chemical process industry and is nowadays the approved method for intelligent control in different domains, including real-time flood control [6-8]. The nonlinear behaviour of river systems during flood events requires nonlinear river models, which turns the problem into a nonlinear programming problem. Such problems are more difficult to solve and can suffer from multiple local optima. Nonlinear MPC can be used to overcome this problem, but is computationally expensive and global optimality is not guaranteed [9-10]. Therefore, Breckpot [11] excluded

\footnotetext{
a Corresponding author: Evert.Vermuyten@kuleuven.be
}

the nonlinear characteristics from the river model to turn the problem into a Quadratic Programming problem. Nevertheless, calculation times were still very long, so this method was therefore only applied so far for small and simple river systems. Another solution is the combination of MPC and a heuristic approach. These heuristic approaches have gained much interest in recent years because of their ability to deal with nonlinearities, multi-objective analyses and uncertainties [12]. The MPC-GA technique is an example of such an approach whereby a Genetic Algorithm (GA) is used in combination with MPC. This technique has already been successfully applied for river flood control [13-15]. In these studies, conceptual models were used in a way complementary to the detailed full hydrodynamic models to limit the model computational times.

The authors have further advanced this technique during recent years. Improvements are: consideration of larger and more complex river networks including floodplains, fast optimization methods that can handle large numbers of optimization variables and total economic flood damage as optimization objective. For that purpose, a Reduced Genetic Algorithm (RGA) was developed, based on the GA [16]. It was concluded that the MPC-RGA technique outperformed the current control strategy and significantly reduced flood consequences. This previous research, however, made the assumption that the model simulations and flood forecasts do not involve uncertainties. This is obviously never the case in practise. Uncertainties can have an important impact on the real-time flood control performance. Because it is expected that the most important source of uncertainty is the rainfall forecast 
uncertainty, this paper investigates the effect of this type of uncertainty on the MPC-RGA performance. Model uncertainty is not considered.

Section 2 gives first an overview of the study area of this research, followed by Section 3, describing the methods. Next, Section 4 discusses the results. Section 5 summarizes the main conclusions of this works and suggestions for future research.

\section{Study Area}

The Demer basin is one of the eleven river basins in Flanders, Belgium. This flood prone basin has suffered from many flood of which the most recent large floods are the ones in January 2002, September 1998 and November 2010. This initial research focuses on the Herk river, which is one of the streams in the Demer basin. In further research, the whole basin will be considered.

The Herk river consists of two streams, the Kleine Herk and the Grote Herk, and eleven floodplains. The flows in the network are controlled by three hydraulic structures. Currently, these hydraulic structures are operated individually by Programmable Logic Controllers (PLCs). The primary task of these PLCs is to protect the city of Stevoort by controlling an inline retention basin, which is located upstream of this city. More details about the Herk river network can be found in Section 4.1

\section{Methods}

\subsection{MPC}

As mentioned above, the hydraulic structures are currently individually operated by means of PLCs. These PLCs have typically an if-then-else structure and take only the current system states into account. This local and instantaneous regulation leads to suboptimal results, since it lacks anticipating competence. By using a process model to predict future system outputs over a certain time horizon, the effect of disturbance variables on these outputs can be taken into account when determining optimal future inputs. This results in a regional, anticipating and dynamic control strategy.

\subsection{MPC-RGA}

Figure 1 gives a schematic overview of the MPCRGA technique, consisting of three building blocks: a river model, a scenario generator and a selector. Each optimization starts with updating the initial model states so that they represent the current system conditions. This is done by eliminating the systematic difference between model results and observations, defined by data assimilation (DA). Next, a possible future control scenario is created by the scenario generator. This gate level (GL) scenario is together with rainfall forecasts applied to the river model. The resulting system state predictions over the control horizon are then compared with the states of the best control strategy so far. The selector selects the best GL scenario with respect to the objective criteria and passes it on to the scenario generator for the next iteration. Because of the specific working principle of MPC-RGA, multiple iterations can be parallelized. When the stopping criterion is met, the first gate levels of the best control scenario are applied to the river system. This whole procedure is repeated at the next control time step.

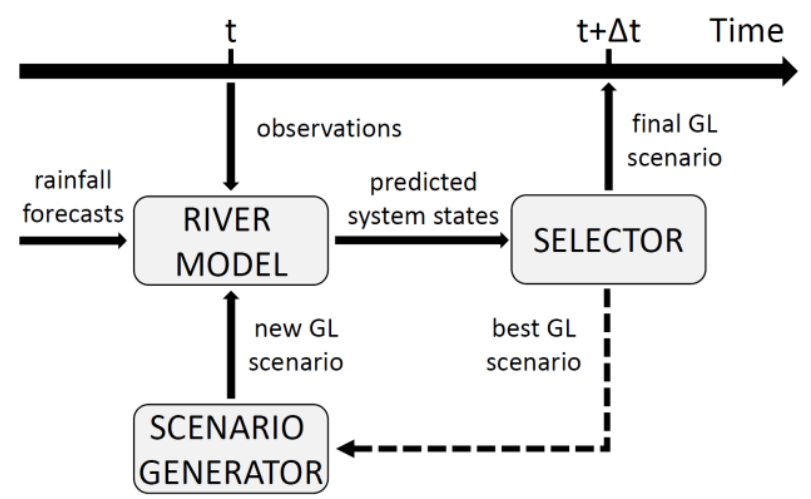

Figure 1. Scheme of the MPC-RGA technique

\subsubsection{Conceptual river model}

Full hydrodynamic river models solve the Saint Venant equations explicitly, which makes them very accurate, but computationally slow. Consistently, these models are not suited for this purpose as many simulations have to be carried out during optimization. Therefore, a conceptual river model is used in this research. Such a reservoir-type model lumps the hydrodynamic processes in space (storage cell concept), resulting in a gain in computational efficiency.

The Conceptual Model Developer tool of Wolfs [17] was used to develop the conceptual model in this work. The modular approach applied in this tool, consists of different model components. These components can be freely combined, depending on the user needs and available data. Although the data-driven calibration of these components, the obtained parameters have a physical meaning. Simulation results of a detailed InfoWorks RS model implemented by the Flemish Environment Agency (VMM) are used as calibration data in this work.

\subsubsection{Scenario generator}

Two different approaches where implemented in the scenario generator to create possible future control strategies. The first approach creates totally new GL scenarios, whereas the second approach mutates the best GL scenario so far.

Totally new GL scenarios are created by generating random gate positions over the control horizon at fixed time steps. By fitting a curve through these positions and the initial gate position, smooth GL series are obtained.

Two options are available for the mutating process: diversification and intensification. Each option starts by 
selecting the gate positions at fixed time steps. The diversification option sets one of these gate positions equal to the previous selected GL. In the intensification option, one of the selected gate positions is shifted a few centimeters up or down. After these mutations, both options fit a new curve through the selected gate positions.

\subsubsection{Selector}

The selector evaluates the different control strategies created by the scenario generator and selects the best strategy with respect to the optimization objectives. Four control priorities are considered in this research: preserving a safety margin for the water level in the retention basin, reducing the catchment-wide economic flood damage cost, preserving a safety margin under critical dikes and restoring storage capacity.

Overtopping of the retention basin dikes could lead to dike failure with huge damage. Therefore, keeping a safety margin for the water level in this basin has the highest priority.

The second priority is to minimize the total flood damage along the river network. For this case study, the International Marine and Dredging Company (IMDC) used the LATIS tool to create economic damage functions which translate the water levels in the floodplains to economic damage costs. This tool was developed by Flanders Hydraulic Research and Ghent University [18] and combines land use maps with socioeconomic data to create maximum damage maps. A relation between the water level and the damage cost for each considered floodplain area is obtained by coupling these maps to damage factors, depending on the type of land use.

Keeping a safety margin for the water levels at critical dikes is the third control priority. Overtopping of these dikes would lead to huge damage, since they are located at damage prone areas.

When all other priorities are met, the storage capacity of the retention basin should be restored as fast as possible to be available for potential new flood events.

\subsection{Rainfall-runoff uncertainty}

In previous research [16], the MPC-RGA technique was tested under the assumption of perfect rainfall forecasts. These forecasts, however, always differ from the real rainfall, resulting in water level predictions deviating from the real future water levels. The MPCRGA algorithm is nevertheless inherently robust against rainfall forecast uncertainties, as feedback about these deviations is received during the model updating phase at every control time step. In order to prove this robustness, the MPC-RGA technique is tested under uncertain rainfall-runoff (RR) discharges forecasts. Since historical rainfall forecasts are not available, the authors decided to artificially add a realistic amount of uncertainty to the actual RR discharges.

RR uncertainty increases along the prediction horizon. Based on the PhD research by Timbe [19] on river flood modelling and uncertainty analysis, we estimated this uncertainty to be around $10 \%$ at the current time step, increased with $0.2 \%$ per hour in the prediction horizon. The same uncertainty estimates were applied by Barjas Blanco [9]. Based on these uncertainty estimates, uncertain RR discharges are generated in the following way:

- At the current time step, a uniformly distributed random error percentage between $-10 \%$ and $10 \%$ is created.

- Then, with fixed time steps, a beta distributed random error percentage between $-p$ and $+p$ is created. The variable $\mathrm{p}$ is defined as $10+0.2 \mathrm{i}$, with $\mathrm{i}$ indicating the time in hours along the prediction horizon. The maximum of this beta distribution is set equal to the previous error percentage and the average to the value corresponding to a fixed percentage between the previous error percentage and 0. By making use of beta distributions, the autocorrelation in the prediction error is taken into account (an overestimation at time $t$ will probably also lead to an overestimation at time $\mathrm{t}+\Delta \mathrm{t})$.

- The generated error percentages are then applied to the actual rainfall at the corresponding time, resulting in uncertain RR discharges.

- A curve is fitted through these error-perturbed discharges versus forecast time, resulting in an uncertain RR forecast.

An example of a generated uncertain RR forecast is shown in Figure 2.

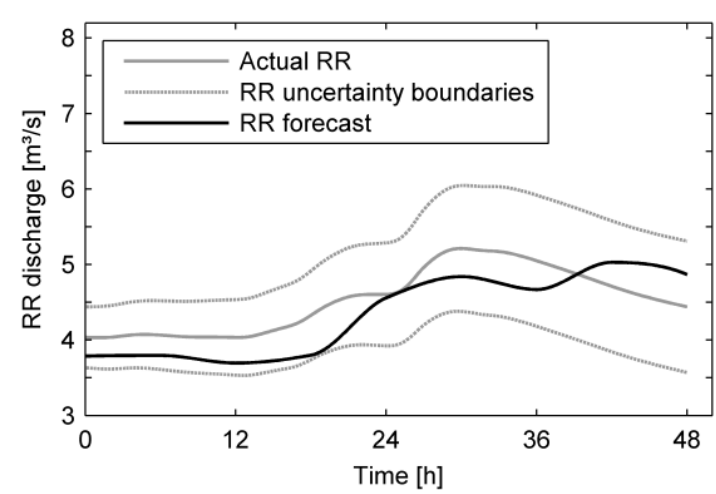

Figure 2. Example of an uncertain RR forecast (black) generated based on the actual RR discharge (grey)

\subsection{Data assimilation}

Data assimilation (DA) is a technique to update model states in real time by using observations of the actual system. As mentioned above, this feedback mechanism makes MPC inherently robust against uncertainties. This research ignores model uncertainty, by making use of the same to model to represent the actual river system (main model) and to make predictions (prediction model). To investigate the influence of rainfall forecast uncertainties, the prediction model uses uncertain $R R$ forecasts 
generated by the method described in section 3.3, while the actual RR discharges are applied to the main model.

The DA method used in this work assumes that each reservoir in the model has water level observations available. Because of this assumption and the fact that the same river model is used as main and prediction model, the initial states of the prediction model can be updated so that they perfectly represent the current state of the main model. This computationally efficient and rather simple DA method gives a first assessment on the added value of DA to reduce performance loss due to rainfall forecast uncertainty. Later, the obtained results can be used as a reference for more realistic DA implementations where observations are not available for every reservoir or to study the benefits of installing new measurement devices.

\subsection{Multiple Model Predictive Control}

Van Overloop proposed the use of Multiple Model Predictive Control (MMPC) to deal with uncertain inflows [20]. This technique minimizes the flood risk by multiplying the control objectives by the probability of occurrence. The MMPC makes use of three different rainfall scenarios: a high, mean and low scenario. Van Overloop successfully applied this technique on a drainage canal system for set-point control. This research investigates if this approach can also improve the realtime flood control performance of the MPC-RGA technique under uncertain rainfall forecasts.

Figure 3 gives a schematic overview of the MMPCRGA technique. This approach is very similar as the MPC-RGA technique, but the generated GL scenarios are now evaluated in three identical, parallel models. The three RR scenarios, created by the uncertainty generator, are applied to this bank of models. The selector analyzes the considered GL scenario with respect to the weighted sum of the optimization criteria for the three RR scenarios.

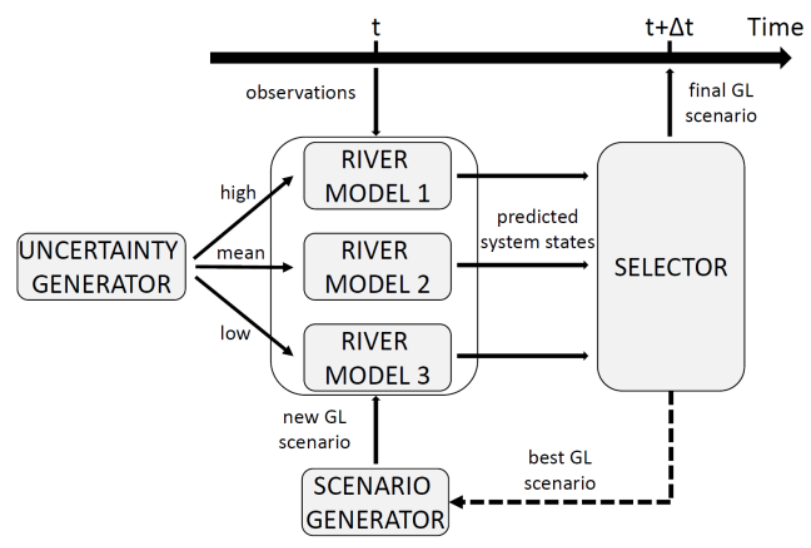

Figure 3. Scheme of the MMPC-RGA technique

To create the three RR scenarios, 50 ensemble RR forecasts are created with the technique described in section 3.3, starting from a deterministic RR forecast. After ranking these ensemble members with respect to the cumulative RR volume, the members are divided into three groups: a high group containing the $10 \%$ highest cumulative volumes, a low group containing the $10 \%$ lowest cumulative volumes and an average group containing the rest of the ensemble members. For each group, the RR values of the different ensemble members are averaged for every time step in the prediction horizon, resulting in a high, mean and low RR scenario. An example of such results is given in Figure 4.

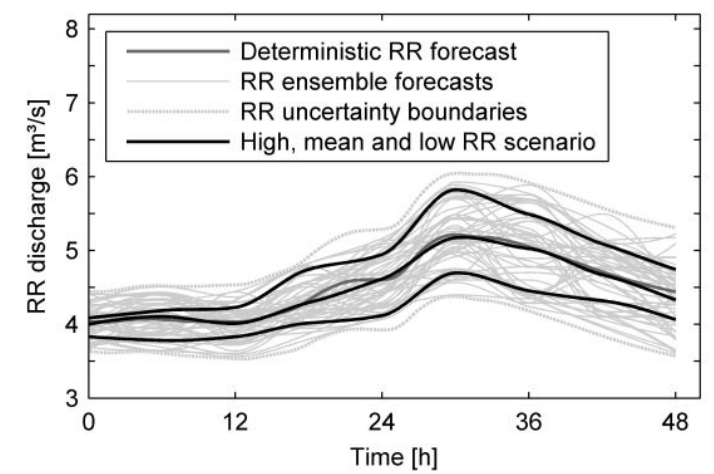

Figure 4. Example of ensemble RR forecasts (light grey) generated based on a deterministic RR prediction (dark grey) and the corresponding high, mean and low RR scenarios (black)

\section{Results}

\subsection{Conceptual model}

The identification, calibration and validation of the conceptual model used in this work is based on the simulation results for ten events obtained with the detailed full hydrodynamic Infoworks RS model of VMM. Both extremely dry and extremely wet periods and artificial as well as historical events are covered by this set of events. The river reaches are conceptualized by means of a series of reservoirs with volume or water level surface profile slope versus throughflow relationships, combined with volume-water level relationships. The floodplains are conceptualized by means of storage cells. These storage cells are integrated in the river reservoirs or merged with each other where possible to gain computational time. A schematic overview of the conceptual model can be found in Figure 5. Reservoirs 10 and 13 (R10 and R13) are the most flood damage prone locations as the city of Stevoort is located in that area. The inline retention basin is modelled by reservoirs 2,9 and 12 (R2, R9 and R12). 


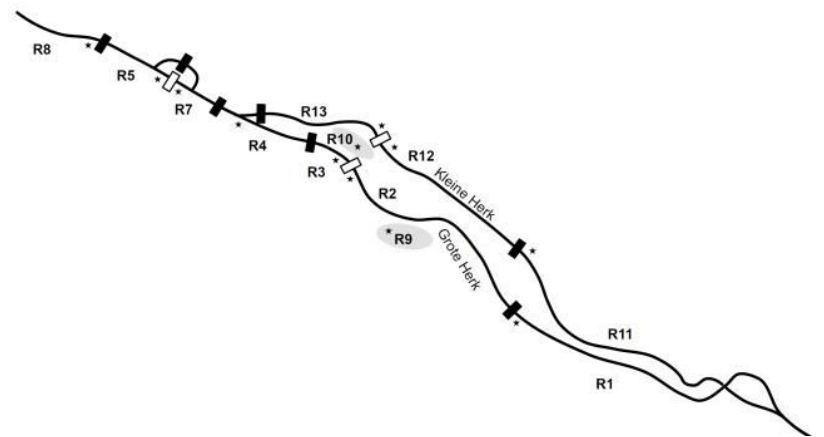

Figure 5. Schematic overview of the conceptual model. The DA updating locations are indicated with stars and the controllable hydraulic structures with hollow boxes.

Three events are selected from the calibration and validation set for this study. Event 1 is the historical event of September 1998, which caused one of the largest floods in the Demer basin. Event 2 is an artificial hydrograph with a return period of 1000 years. Event 3 is again an artificial event, which consists of two times the historical event of September 1998 in a row.

\subsection{RR uncertainty analysis}

Given the main aim of this research, to quantify the loss in performance of the MPC-RGA technique due to rainfall forecast uncertainty, three control strategies are considered for this quantification. These are the current PLC regulation, MPC-RGA with perfect RR forecasts (MPC-PF) and MPC-RGA with uncertain RR forecast created by the method described in section 3.3 (MPCUF). For both MPC strategies, data assimilation is used to initialize the prediction model states. Figure 6 summarizes the obtained results for the three control strategies. Averaged costs from different optimization runs are used to exclude the variability in the MPC-RGA results.

Obviously, the performance of the MPC-RGA technique worsens when uncertain RR forecasts are considered. Nevertheless, MPC-RGA still outperforms the current PLC control strategy. At first sight, MPC-UF has no clear advantage for event 1 in comparison to the PLC regulation. A more detailed analysis of the results shows however that the retention basin dike criterion is violated in the PLC control strategy, while MPC-UF succeeds in keeping this safety margin under the retention basin dike. This means that even by using less storage capacity, MPC performs slightly better than the PLC strategy. For events 2 and 3, the loss in performance due to the introduction of RR uncertainty is limited to respectively $8 \%$ and $2 \%$. When comparing the optimization results of MPC-PF and MPC-UF only small differences in the control strategy are noticed. These results prove the inherent robustness of MPC-RGA against rainfall forecast uncertainties.

To assess the MPC-RGA performance loss under worst case conditions, the previous test is repeated for RR forecasts that are consistently under- or overestimated. These forecasts are generated by creating 50 ensemble $\mathrm{RR}$ forecasts based on the actual RR discharge and extracting a high and low scenario from these ensembles, similar as for MMPC (section 3.5). The resulting performance loss is $15 \%$ for a consistent overestimation of the RR discharge and $13 \%$ for a consistent underestimation.

Figures 7, 8 and 9 show more detailed results for MPC-RGA optimizations with perfect forecasts (MPC$\mathrm{PF}$ ), consistently overestimating RR forecasts (MPC$\mathrm{OEF}$ ) and consistently underestimating $\mathrm{RR}$ forecasts (MPC-UEF). The results are presented for the total flood damage along the river system (Figure 7), the water level in the retention basin (Figure 8) and the gate position of the hydraulic structure in the Kleine Herk, which is the most important structure to control the retention basin (Figure 9).

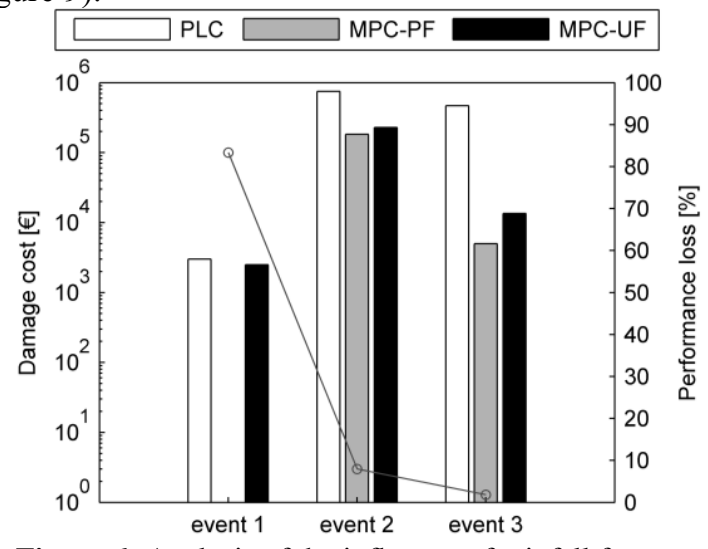

Figure 6: Analysis of the influence of rainfall forecast uncertainty on the MPC-RGA performance

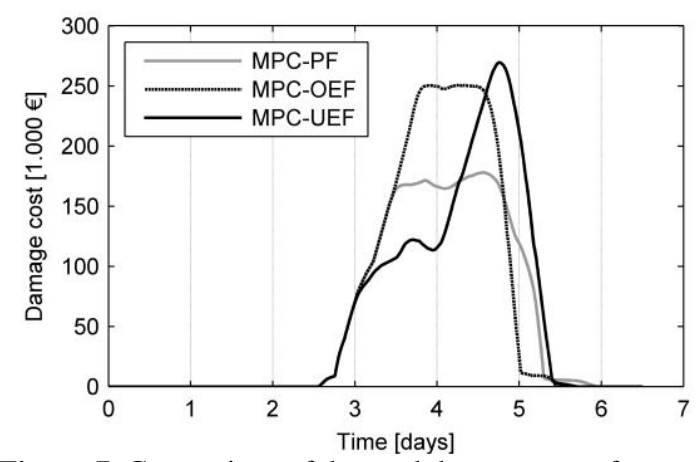

Figure 7. Comparison of the total damage cost after applying MPC-RGA for perfect RR forecasts (grey), consistently overestimating RR forecasts (dashed black) and consistently underestimating RR forecasts (black).

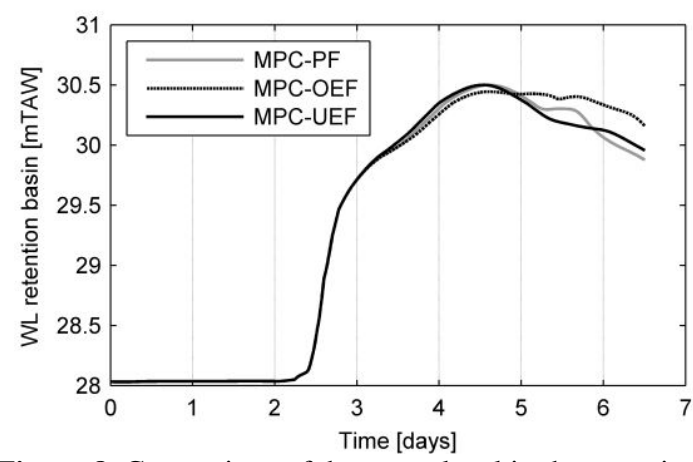

Figure 8. Comparison of the water level in the retention basin after applying MPC-RGA for perfect RR forecasts (grey), consistently overestimating RR forecasts (dashed black) and consistently underestimating RR forecasts (black). 


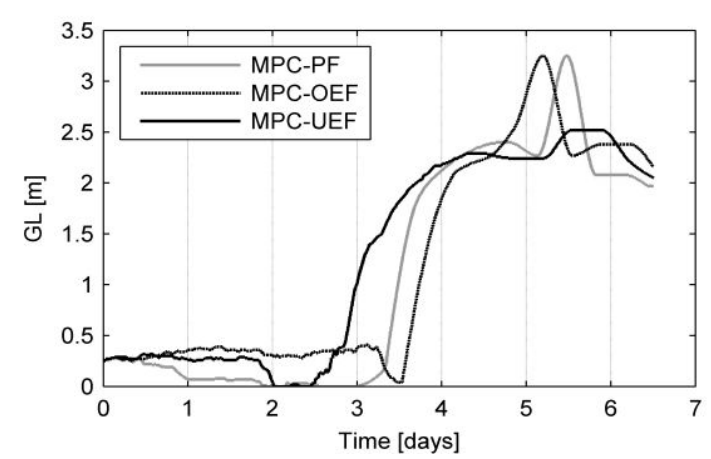

Figure 9. Comparison of the gate position of the hydraulic structure in the Kleine Herk after applying MPC-RGA for perfect RR forecasts (grey), consistently overestimating RR forecasts (dashed black) and consistently underestimating RR forecasts (black).

When the RR forecasts are consistently underestimating the real $\mathrm{RR}$ values, this results in a too early filling of the retention basin. As seen in Figure 9, the gate position of the hydraulic structure in the Kleine Herk rises much earlier in comparison to the PF scenario. Because of this, the incurred damage cost is lower halfway the event. The available storage capacity is, however, used too early, leading to a peak in the flood damage in the second phase of the event.

When the RR forecasts are consistently overestimated, the opposite effect is noted: the gate raising goes too late, resulting in a later filling of the retention basin. Besides, as the RR forecasts overestimate the future rainfall volume, the MPC-RGA algorithm makes storage capacity available for later use. Because the actual future rainfall volumes are lower than the forecasted ones, part of the storage capacity is unused. This suboptimal control strategy leads to a higher flood damage cost, as shown in Figure 7.

\subsection{Multi Model Predictive Control analysis}

In order to reduce the loss in performance due to rainfall forecast uncertainties, Multiple Model Predictive Control (MMPC) was tested for three RR forecast scenarios. The obtained results are compared with optimization results making use of uncertain deterministic RR forecasts (MPC-DF). These deterministic forecasts are generated by the ensemble method described in section 3.5, starting from the actual RR discharges. The low RR scenario created in this way leads to consequently underestimating $R R$ forecasts (UEF), the high RR scenario to consequently overestimating $\mathrm{RR}$ forecasts (OEF) and the mean scenario to accurate, but slightly uncertain, RR forecasts (AUF). The three RR scenarios used by MMPC are derived from these uncertain deterministic RR forecasts, as described in section 3.5. Table 1 summarizes the obtained results.

\begin{tabular}{|c|c|c|c|c|}
\hline \multicolumn{2}{|c|}{ Damage cost $[\boldsymbol{\epsilon}]$} & OEF & AUF & UEF \\
\hline \multirow{2}{*}{ Event 1} & MPC-DF & 300 & 1000 & 1300 \\
\cline { 2 - 5 } & MMPC & 2700 & 500 & 800 \\
\hline
\end{tabular}

\begin{tabular}{|c|c|c|c|c|}
\hline \multirow{2}{*}{ Event 2 } & MPC-DF & 265000 & 195000 & 240000 \\
\cline { 2 - 5 } & MMPC & 300000 & 240000 & 190000 \\
\hline \multirow{2}{*}{ Event 3 } & MPC-DF & 12000 & 6600 & 5000 \\
\cline { 2 - 5 } & MMPC & 24000 & 7000 & 6500 \\
\hline
\end{tabular}

Table 1. Comparison of the damage cost between MPC with deterministic uncertain inflows (MPC-DF) and MMPC for consistently overestimating RR forecasts (OEF), consistently underestimating RR forecasts (UEF) and accurate uncertain forecasts (AUF).

For event 1, MMPC performs in general slightly better than MPC-RGA with uncertain deterministic RR forecasts, while it performs slightly worse for event 3 . The damage costs of these events are however small and differences between the two approaches are limited. For the most extreme event 2, MMPC shows a large improvement for underestimated rainfall forecasts, but a huge decline in performance for the other two rainfall scenarios. This improvement for the underestimated rainfall scenario can be assigned to the fact that the high RR scenario in MMPC lies very close to the actual RR discharge. As this scenario fully determines the optimization to avoid crossing the safety level in the retention basin in all possible RR scenarios, an almost optimal control strategy with respect to the actual rainfall is achieved. The high RR scenario will, however, also determine the optimizations for the other two rainfall scenarios (OEF and AUF). This leads to a control strategy leaving part of the storage capacity of the retention basin unused as the algorithm overestimates future rainfall volumes, similar to what was explained in section 4.2. Consequently, the damage cost with MMPC is higher for these two rainfall scenarios than for the deterministic MPC result. MMPC especially performs worse for all three events in case of overestimated rainfall. This is not surprising, as the high RR scenario fully determines the MMPC optimization, doubles this overestimation and the storage capacity of the retention basin remains underutilized for the actual rainfall.

Despite the introduction of forecast uncertainties, both optimization methods succeed in keeping a safety margin under the retention basin dike crest for all three rainfall scenarios. In case this criterion is violated, the exceedance of the safety level is limited to $0.5 \mathrm{~mm}$, as a result of the receding horizon strategy of MPC.

\section{Conclusions}

Previous research has shown that intelligent real-time control of hydraulic structures is very efficient for flood control under uncertainty-free conditions. The presence of model and rainfall forecast uncertainties can, however, significantly affect the performance of flood control strategies. This paper quantified the control performance loss due to rainfall forecast uncertainties and demonstrated that the MPC-RGA results are inherently robust against these uncertainties.

To quantify the loss in performance, uncertain rainfall-runoff forecasts were created by adding a realistic 
amount of uncertainty to the actual rainfall-runoff discharges. The results obtained with these uncertain rainfall-runoff forecasts were compared with the results of the PLC regulation and MPC-RGA results for perfect rainfall forecasts. Despite the introduction of rainfall forecast uncertainties, the MPC-RGA approach still outperformed the current PLC regulation. Obviously, the control performance deteriorated in comparison to MPCRGA with perfect rainfall forecasts. The loss in control performance is, however, less than $10 \%$. Under worstcase conditions, consisting of consistently under- or overestimating rainfall-runoff forecasts, the performance loss increased to a maximum of $15 \%$. The low performance losses, even under extreme conditions, demonstrate the inherent robustness of MPC-RGA against rainfall forecast uncertainties due to its receding horizon strategy.

Next, Multiple Model Predictive Control was tested in order to reduce the performance loss due to rainfall forecast uncertainties. This method introduces high, mean and low rainfall runoff forecast scenarios, which are then simultaneously optimized. In general, MMPC does not show a clear benefit for flood control in comparison to the deterministic MPC. For small flood events, both methods perform similar, but for extreme flood events, MMPC worsens the optimization results. The reason for this is the high scenario, which fully determines the optimizations with respect to the safety level criterion in the retention basin. As a consequence, MMPC underutilises the available storage capacity, especially for overestimating rainfall forecasts.

Another possible approach to reduce the performance loss due to uncertainties consists of a more sophisticated data assimilation method. This method provides feedback about the states of the river system, including the actual rainfall runoff discharges, to the optimization algorithm. In this way, rainfall-runoff forecasts can be corrected by taking forecast errors at previous time steps into account. This approach will be investigated in further research.

\section{Acknowledgments}

This research was supported by the Agency for Innovation by Science and Technology in Flanders (IWT). The authors would like to thank Innovyze for the InfoWorks software license, and the Flemish Environment Agency (VMM) for the data and InfoWorks RS model of the Demer basin.

\section{References}

1. HIC (2003). The Digital Demer: a new and powerful instrument for water level management (in Dutch). Hydrologic information service of the authorities of Flanders, Borgerhout, Belgium, 28 p.

2. IPCC (2014). Climate Change 2014: Impacts, Adaptation, and Vulnerability. Summary for Policymakers, Working Group II contribution to the Fifth Assessment Report of the Intergovernmental Panel on Climate Change, $44 \mathrm{p}$.
3. Willems P., Olsson J., Arnbjerg-Nielsen K., Beecham S., Pathirana A., Bülow Gregersen I., Madsen H. and Nguyen V.-T.-V. (2012). Impacts of Climate Change on Rainfall Extremes and Urban Drainage. IWA Publishing, 252 p., Paperback Print ISBN 9781780401256; Ebook ISBN 9781780401263.

4. Vansteenkiste T., Tavakoli T., Ntegeka V., De Smedt F., Batelaan O., Pereira F. and Willems P. (2014) Intercomparison of hydrological model structures and calibration approaches in climate scenario impact projections. Journal of Hydrology, 519, 743755.

5. Poelmans L., Van Rompaey A., Ntegeka V. and Willems P. (2011). The relative impact of climate change and urban expansion on river flows: a case study in central Belgium. Hydrological Processes, 25(18), 2846-2858.

6. Wendt M., Li P. and Wozny G. (2002). Nonlinear chance-constrained process optimization under uncertainty. Industrial and Engineering Chemistry Research, 41(15), 3621-3629.

7. Nagy Z.K. (2009). Model based robust control approach for batch crystallization product design. Computers and Chemical Engineering, 33, 16851691.

8. Qin S. and Badgwell T. (2003). A survey of industrial model predictive control technology. Control Engineering Practice, 11(7), 733-764.

9. Barjas Blanco T., Chiang P., Breckpot M., Willems P., Berlamont J. and De Moor B. (2010). Flood regulation using non-linear model predictive control. Control Engineering Practice, 18(10), 1147-1157.

10. Schwanenberg D., Verhoeven G., Van Den Boogaard H. and van Overloop P.-J. (2010). Nonlinear model predictive control of flood detention basins in operational flood forecasting. 9th International Conference on Hydroinformatics, New York City, USA, 17-21 August 2014.

11. Breckpot M., Agudelo O.M., Meert P., Willems P. and De Mooi B. (2013). Flood control of the Demer by using Model Predictive Control. Control Engineering Practice, 21(12), 1776-1787.

12. Rani D. and Moreira M.M. (2010). SimulationOptimization Modeling: A survey and Potential Application in Reservoir Systems Operation. Water Resources Management, 24(6), 1107-1138.

13. Van den Zegel B., Vermuyten E., Wolfs V., Meert P. and Willems P. (2014). Real-time control of floods along the Demer river, Belgium, by means of MPC in combination with GA and a fast conceptual river model. 11th International Conference on Hydroinformatics, New York City, USA, 17-21 August 2014.

14. Vermuyten E., Van den Zegel B., Wolfs V., Meert P. and Willems P. (2014). Real-time flood control by means of an improved MPC-GA algorithm and a fast conceptual river model for the Demer basin in Belgium. 6th International Conference on Flood Management, Sao Paulo, Brazil, 16-18 September 2014. 
15. Chiang P. and Willems P. (2015). Combine evolutionary optimization with Model Predictive Control in real-time flood control of a river system. Water Resources Management, 29(8), 2527-2542.

16. Vermuyten E., Meert P., Wolfs V. and Willems P. (2015). Using a fast conceptual river model for floodplain inundation forecasting and real-time flood control - a case study in Flanders, Belgium. 21st International Congress on Modeling and Simulation, Broacbeach, Queensland, Australia, 29 November 4 December 2015.

17. Wolfs V., Meert P. and Willems P. (2015). Modular conceptual modelling approach and software for river hydraulic simulations. Environmental Modelling \& Software, 71, 60-77.

18. Kellens W., Deckers P., Saleh H., Vanneuville W., De Maeyer P., Allaert G. and De Sutter R. (2008). A GIS tool for flood risk analysis in Flanders (Belgium). In C. Brebbia \& E. Beriatos (eds.), Risk analysis VI: computer simulation and hazard mitigation: 21-27. Presented at the 6th International conference on Computer Simulation Risk Analysis and Hazard Mitigation, Southampton, UK: WIT Press.

19. Timbe L. (2007). River flooding analysis using quasi-2D hydraulic modelling and geospatial data. Ph.D. thesis, Faculty of Engineering, KU Leuven, Leuven, Belgium.

20. Van Overloop P.-J., Weijs S. and Dijkstra S. (2008). Multiple model predictive control on a drainage canal system. Control Engineering Practice, 16(5), 531-540. 